\title{
Angiogenic and Angiostatic Chemokines in Idiopathic Pulmonary Fibrosis and Granulomatous Lung Disease
}

\author{
Ai Cui ${ }^{a, f}$ Olaf Anhenn ${ }^{a, b}$ Dirk Theegarten ${ }^{b}$ Shinichiro Ohshimo ${ }^{a}$ \\ Francesco Bonella ${ }^{a}$ Stephan U. Sixt ${ }^{c}$ Juergen Peters ${ }^{c}$ Rafael Sarria ${ }^{e}$ \\ Josune Guzman ${ }^{d}$ Ulrich Costabel ${ }^{a}$ \\ a Pneumology and Allergology, Ruhrlandklinik, Medical Faculty, University of Duisburg-Essen, ${ }^{b}$ Institute of \\ Pathology and Neuropathology and ${ }^{\mathrm{C}}$ Anesthesiology and Intensive Care Medicine, University Hospital, Essen,

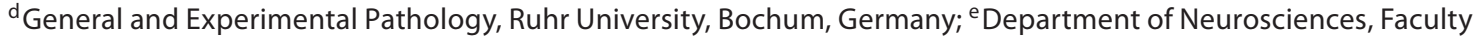 \\ of Medicine and Dentistry, Basque Country University, Bilbao, Spain; ${ }^{f}$ Beijing Institute of Respiratory Medicine, \\ Beijing Chaoyang Hospital, Capital University of Medical Sciences, Beijing, China
}

\section{Key Words}

Alveolar macrophage $\cdot$ Chemokine $\cdot$ Granulomatous lung disease $\cdot$ Idiopathic pulmonary fibrosis

\begin{abstract}
Background: Angiogenesis-angiostasis balance and leukocyte recruitment are influenced by different concentrations of distinct chemokines. Objective: To investigate the relative contribution of angiogenic and angiostatic CXC chemokines to the pathogenesis of idiopathic pulmonary fibrosis (IPF) and granulomatous lung diseases, we examined the in vitro production of an angiogenic chemokine (IL-8), and 2 angiostatic chemokines (IP-10 and MIG) by alveolar macrophages. Methods: Alveolar macrophages from 16 patients with granulomatous lung diseases [8 with sarcoidosis, 8 with extrinsic allergic alveolitis (EAA)], 16 patients with IPF, and 8 control subjects were cultured for $24 \mathrm{~h}$. IL-8, IL-18, IP-10 and MIG in the culture supernatants were measured by a fluorescent bead-based multiplex technique. Results: In IPF patients, IL8 was increased and correlated with bronchoalveolar lavage (BAL) neutrophils, whereas the levels of IP-10 and MIG were
\end{abstract}

normal. In sarcoidosis and EAA patients, IL-8, IP-10, and MIG were all increased and IP-10 and MIG correlated with IL-18, a Th1 cytokine, and the percentage and number of BAL lymphocytes. Conclusions: The difference in the expression of CXC chemokines and a Th1 cytokine may contribute to the different immunopathogenesis, clinical course and responsiveness to treatment of these diseases.

Copyright $\odot 2009$ S. Karger AG, Basel

\section{Introduction}

Interstitial lung diseases are a heterogeneous group of disorders including idiopathic pulmonary fibrosis (IPF) and granulomatous interstitial lung diseases. IPF is a chronic and fatal lung disease of unknown etiology, characterized by progressive fibroblast proliferation and fibrosis, destruction of the alveolar architecture, and a relentless decline in pulmonary function [1]. Sarcoidosis is a multisystem immunologic disorder of unknown etiology, characterized initially by a T-helper (Th) lymphocyte/macrophage alveolitis and later by the formation of

\section{KARGER}

Fax +41613061234 E-Mail karger@karger.ch www.karger.com (c) 2009 S. Karger AG, Basel

0025-7931/10/0805-0372\$26.00/0

Accessible online at:

www.karger.com/res
Ulrich Costabel, MD

Ruhrlandklinik, Tueschener Weg 40

DE-45239 Essen (Germany)

Tel. +49 201433 4020, Fax +492014334029

E-Mail ulrich.costabel@ruhrlandklinik.de 
Table 1. Demographics

\begin{tabular}{lccc}
\hline & IPF & $\begin{array}{l}\text { Sarcoidosis } \\
\text { and EAA }\end{array}$ & Controls \\
\hline Female:male, $n$ & $2: 14$ & $7: 9$ & $3: 5$ \\
Age (mean \pm SEM), years & $66 \pm 2$ & $49 \pm 3$ & $29 \pm 2$ \\
Smoker (non:ex:current), $\mathrm{n}$ & $6: 9: 1$ & $14: 2: 0$ & $8: 0: 0$ \\
\hline
\end{tabular}

epithelioid cell granulomas [2]. Extrinsic allergic alveolitis (EAA), also called hypersensitivity pneumonitis, is caused by repeated inhalation of and sensitization to finely dispersed antigens, and is characterized by an alveolitis composed of activated lymphocytes and macrophages, and by epithelioid cell granuloma formation [3]. Although some patients with sarcoidosis or EAA can develop lung fibrosis at the later stage of disease, they usually show a much better clinical course and are more responsive to corticosteroid treatment than patients with IPF.

Angiogenesis, defined as the process of new blood vessel growth, is an important component of wound healing [4] and may contribute to fibroproliferation and extracellular matrix deposition. Studies have demonstrated the presence of angiogenic factors, such as epithelial neutrophil-activating peptide 78, in IPF patients [5] and implicated the new formation of blood vessels as an important aspect in bleomycin-induced lung fibrosis in rats [6]. Angiogenesis-angiostasis balance and leukocyte recruitment are influenced by different concentrations of distinct chemokines. Based on the number and the location of cysteine residues, chemokines are separated into 4 groups: $\mathrm{C}, \mathrm{CC}, \mathrm{CXC}$ and $\mathrm{CX}_{3} \mathrm{C}$ chemokines [7]. CXC chemokines, which contain cysteine residues separated by a single a mino acid, have been demonstrated to be involved in the adjustment of the angiogenesis-angiostasis balance [8]. They also function as potent mediators of inflammation due to their ability to recruit and activate specific leukocytes [9]. Based on their molecular structure [whether an internal glutamate-leucine-arginine (ELR) motif is present or not], CXC chemokines can be further divided into 2 subgroups: ELR+ CXC and ELR- CXC chemokines. Each subgroup exerts different effects on angiogenesis and leukocyte recruitment. For example, ELR+ $\mathrm{CXC}$ chemokines have been demonstrated to be involved in angiogenesis, whereas ELR- CXC chemokines behave as potential inhibitors of angiogenesis [8]. The production of ELR- CXC chemokines can be induced by IFN- $\gamma$
[10]. IL-12 and IL-18, two Th1 cytokines with IFN- $\gamma$ inducing capacity, have been shown to inhibit angiogenesis through up-regulation of ELR- CXC chemokines in mice [11].

To investigate the relative contribution of ELR+ and ELR- CXC chemokines to the pathogenesis of IPF and granulomatous lung diseases, we here examined the in vitro production of an ELR+ chemokine (IL-8), two ELRchemokines (MIG and IP-10), and IL-18 by alveolar macrophages (AM).

\section{Materials and Methods}

\section{Study Population}

Consecutive patients with IPF $(n=16)$ or granulomatous lung diseases ( 8 with chronic EAA, and 8 with active pulmonary sarcoidosis) and healthy volunteers $(n=8)$ were investigated (table 1$)$. The subjects were studied at the time of diagnosis. No subject was under treatment with corticosteroids and/or immunosuppressants at the time of bronchoalveolar lavage (BAL).

IPF patients were diagnosed according to the American Thoracic Society/European Respiratory Society criteria, including the high-resolution tomography characteristics [12]. No patient had left ventricular cardiac failure or history of chronic pulmonary infections. Sarcoidosis patients were diagnosed on the basis of compatible clinical and radiographic features, histological evidence of noncaseating granulomas on transbronchial biopsy or an increased CD4/CD8 ratio in BAL fluid, and the exclusion of other granulomatous lung diseases [2]. Criteria of disease activity, which were fulfilled by all patients, were: (1) recently developed or increasing symptoms such as cough, dyspnea, weakness, fever and arthralgia, and/or (2) chest radiographic evidence of progressive disease, and/or (3) deterioration of lung function tests. According to chest radiographic staging, 7 had stage II, and 1 had stage III. EAA patients fulfilled the following diagnostic criteria: (1) a history of exposure to organic antigens; (2) clinical signs and symptoms consistent with EAA; (3) radiological features and/or functional abnormalities characteristic of interstitial lung disease; (4) evidence of serum precipitins against 1 or more organic antigens, and (5) BAL fluid with increased lymphocytes.

Written informed consent was obtained from all subjects according to institutional guidelines. The study was approved by the local institutional review board.

\section{BAL Procedure}

BAL was performed by instilling sterile isotonic saline into the right middle or left lingular lobe in 5-10 $\times 20 \mathrm{ml}$ aliquots to a total volume of $100-200 \mathrm{ml}$ via a fiber optic bronchoscope with immediate aspiration by gentle suction after each aliquot. A volume of greater than 50\% was retrieved. The recovered BAL fluid was filtered through 2 layers of sterile gauze and subsequently centrifuged at $500 \mathrm{~g}$ for $10 \mathrm{~min}$ at $4^{\circ} \mathrm{C}$. The cells were counted in a hemocytometer. Cell viability was assessed by trypan blue exclusion. Cell differentials were made on smears stained with MayGrünwald-Giemsa by counting 600 cells. 
Table 2. Differential cell profiles in BAL fluid

\begin{tabular}{|c|c|c|c|}
\hline & $\begin{array}{l}\text { IPF } \\
(n=16)\end{array}$ & $\begin{array}{l}\text { Sarcoidosis } \\
\text { and EAA } \\
(\mathrm{n}=16)\end{array}$ & $\begin{array}{l}\text { Controls } \\
(\mathrm{n}=8)\end{array}$ \\
\hline & \multicolumn{2}{|c|}{ Macrophages } & $7.1 \pm 0.5$ \\
\hline$\%$ & $77.5 \pm 3.4^{*}$ & $47.1 \pm 5.4^{* * *, \dagger \dagger \dagger}$ & $88.6 \pm 3.2$ \\
\hline $\mathrm{n} \times 10^{4} / \mathrm{ml}$ & $17.2 \pm 3.1^{* *}$ & $11.9 \pm 2.3^{*}$ & $6.6 \pm 0.6$ \\
\hline \multicolumn{4}{|l|}{ Lymphocytes } \\
\hline$\%$ & $7.4 \pm 0.8$ & $47.6 \pm 5.2^{* * *, \dagger \dagger \dagger}$ & $10.3 \pm 3.2$ \\
\hline $\mathrm{n} \times 10^{4} / \mathrm{ml}$ & $1.6 \pm 0.3^{*}$ & $13.1 \pm 2.3^{* * *, \dagger \dagger}$ & $0.7 \pm 0.2$ \\
\hline \multicolumn{4}{|l|}{ Neutrophils } \\
\hline$\%$ & $10.3 \pm 3.2^{* * *}$ & $3.0 \pm 0.9^{\dagger \dagger}$ & $1.1 \pm 0.4$ \\
\hline $\mathrm{n} \times 10^{4} / \mathrm{ml}$ & $2.4 \pm 0.8^{* * *}$ & $0.9 \pm 0.3^{*}$ & $0.1 \pm 0.0$ \\
\hline \multicolumn{4}{|l|}{ Eosinophils } \\
\hline$\%$ & $5.0 \pm 1.4^{* *}$ & $1.7 \pm 0.7^{\dagger}$ & $0.1 \pm 0.0$ \\
\hline $\mathrm{n} \times 10^{4} / \mathrm{ml}$ & $0.9 \pm 0.3^{* *}$ & $0.5 \pm 0.2$ & $0.0 \pm 0.0$ \\
\hline
\end{tabular}

Values are mean \pm SEM.

${ }^{*} \mathrm{p}<0.05 ;{ }^{* *} \mathrm{p}<0.01 ;{ }^{* * *} \mathrm{p}<0.001$, all vs. controls.

${ }^{\dagger} \mathrm{p}<0.05 ;{ }^{\dagger \dagger} \mathrm{p}<0.01 ;{ }^{\dagger \dagger} \mathrm{p}<0.001$, all vs. IPF.

\section{Cell Culture}

AM cultures were performed as previously described [13]. The cells were washed 3 times with PBS and then resuspended to a final concentration of $1 \times 10^{6}$ cells/ml in RPMI 1640 medium supplemented with $10 \%$ heat-inactivated FCS, $2 \mathrm{mM}$ L-glutamine, $200 \mathrm{U} / \mathrm{ml}$ penicillin and $200 \mu \mathrm{g} / \mathrm{ml}$ streptomycin (Seromed; Biochrom KG, Berlin, Germany). The cell suspension was added at $1 \times 10^{6}$ cells/well to a 24 -well plastic tissue culture plate (Falcon; BD, Franklin Lakes, N.J., USA) and incubated at $37^{\circ} \mathrm{C}$ in a $5 \% \mathrm{CO}_{2}$ humidified atmosphere for $1 \mathrm{~h}$ to permit the adherence of AM. The nonadherent cells were removed by 3 washes with culture medium. The purity of adherent AM was identified to be greater than $95 \%$ by morphology and nonspecific esterase staining. The purified AM were incubated for an additional $24 \mathrm{~h}$ with $1 \mathrm{ml} 10 \%$ RPMI alone or with $1 \mathrm{ml} \mathrm{10 \%} \mathrm{RPMI} \mathrm{and} 100 \mathrm{ng} / \mathrm{ml}$ LPS (SigmaAldrich, St. Louis, Mo., USA). The culture supernatants were harvested and centrifuged, then stored in fractions at $-80^{\circ} \mathrm{C}$ until analysis.

\section{Assays for IL-8, MIG, IP-10 and IL-18}

The concentrations of IL-8, MIG, IP-10, and IL-18 in cultured supernatants were measured by a special fluorescent bead-based multiplex technique (Bio-Rad BioPlex 200; Bio-Rad Laboratories, Munich, Germany). The assessments were performed according to the manufacturer's instructions. The concentrations were expressed as $\mathrm{pg} / \mathrm{ml} \times 10^{6} \mathrm{AM}$ after correction for the proportion of AM.

\section{Statistical Analysis}

Data are expressed as mean \pm SEM. Groups were compared by Student's t test or 1-way ANOVA. The correlation of different parameters was analyzed by Spearman rank. A $p<0.05$ was accepted as statistically significant.

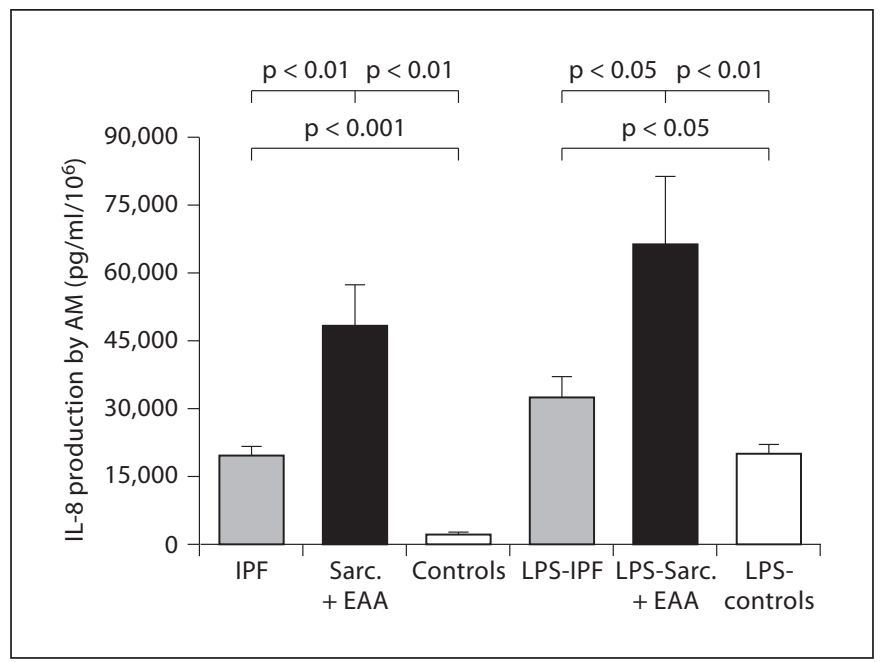

Fig. 1. Release of IL- 8 by AM cultured in the absence or presence of LPS. Data are mean \pm SEM. Sarc. $=$ Sarcoidosis.

\section{Results}

\section{$B A L$ Cell Differentials}

As shown in table 2, patients with IPF had significantly increased percentage and number of neutrophils $(\mathrm{p}<$ 0.001 ) and eosinophils ( $p<0.01)$ compared to controls. Patients with sarcoidosis and EAA had significant increases of lymphocytes compared to controls and IPF ( $\mathrm{p}<0.001$, respectively).

\section{IL-8/CXCL8 Levels Are Elevated in Both IPF and Granulomatous Lung Diseases}

As shown in figure 1 , after $24 \mathrm{~h}$ of culture, the spontaneous in vitro production of IL-8 from AM was significantly higher in IPF and granulomatous lung diseases than in controls ( $\mathrm{p}<0.0001, \mathrm{p}<0.01$, respectively). The LPS-stimulated production of IL- 8 was also significantly higher in IPF and granulomatous lung diseases $(\mathrm{p}<0.05)$ than in controls. The IL-8 production was lower in IPF than in granulomatous lung diseases, with and without LPS stimulation ( $\mathrm{p}<0.05, \mathrm{p}<0.01$, respectively).

\section{MIG/CXCL9 and IP-10/CXCL10 Levels Are Elevated in Granulomatous Lung Diseases but Not in IPF}

The production of MIG from AM was significantly higher in granulomatous lung diseases than in IPF or controls in the absence ( $\mathrm{p}<0.001, \mathrm{p}<0.01$, respectively) or presence of LPS stimulation $(p<0.01)$. The spontaneous production of IP-10 from AM was significantly increased in granulomatous lung diseases compared to IPF 


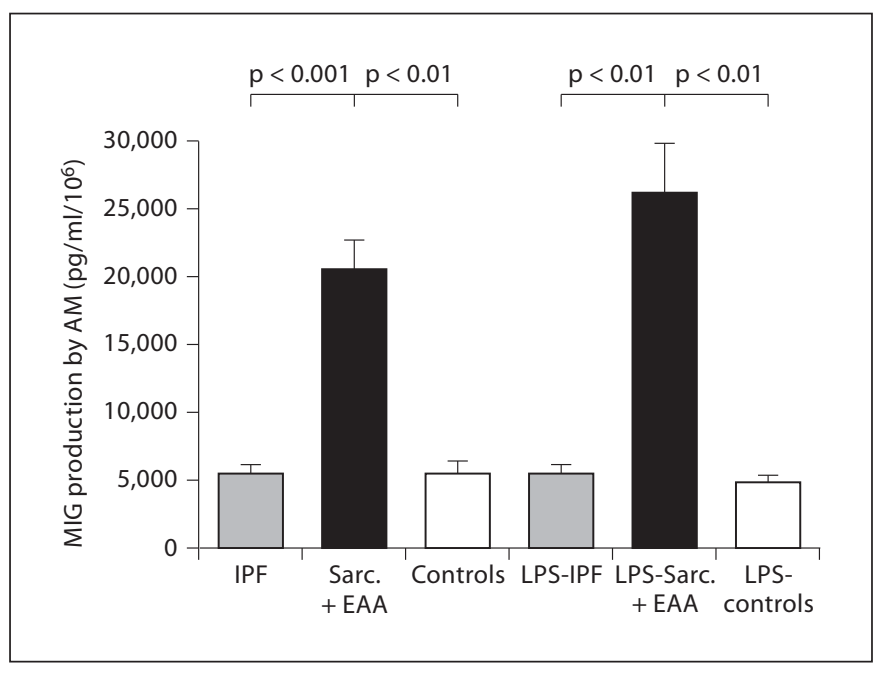

Fig. 2. The release of MIG/CXCL9 by AM cultured in the absence or presence of LPS stimulation. Data are mean \pm SEM. Sarc. $=$ Sarcoidosis.

patients and controls $(\mathrm{p}<0.05)$. With LPS stimulation the IP-10 production was also significantly increased in granulomatous lung diseases compared to IPF or controls $(\mathrm{p}<0.01, \mathrm{p}<0.05$, respectively). There were no differences in the levels of MIG and IP-10 between IPF and controls (fig. 2, 3).

\section{IL-18 Levels Are Elevated in Granulomatous Lung}

\section{Diseases but Not in IPF}

The levels of IL-18 produced by AM were significantly increased in granulomatous lung diseases, but not in IPF, compared with controls, both with $(\mathrm{p}<0.001, \mathrm{p}<0.05$, respectively) and without LPS stimulation $(\mathrm{p}<0.01$, $\mathrm{p}<$ 0.01 , respectively; fig. 4).

\section{Correlation between Chemokine and IL-18 Production} by $A M$

In sarcoidosis and EAA patients, there was a positive correlation between IL-18 and MIG $(r=0.6566, p<0.05)$ or IP-10 ( $\mathrm{r}=0.5658, \mathrm{p}<0.05$; fig. 5). In IPF patients, no correlation was found between IL-18 and MIG or IP-10.

\section{Correlation between Chemokine Production by AM} and BAL Cell Differentials

In IPF patients, there was a significant correlation between IL- 8 and the percentage or number of neutrophils in the BAL fluid $(\mathrm{r}=0.5615, \mathrm{p}<0.05 ; \mathrm{r}=0.5559, \mathrm{p}<0.05$, respectively; fig. 6 ), and no other correlations between IL-8 and BAL cell types. In granulomatous lung diseases,

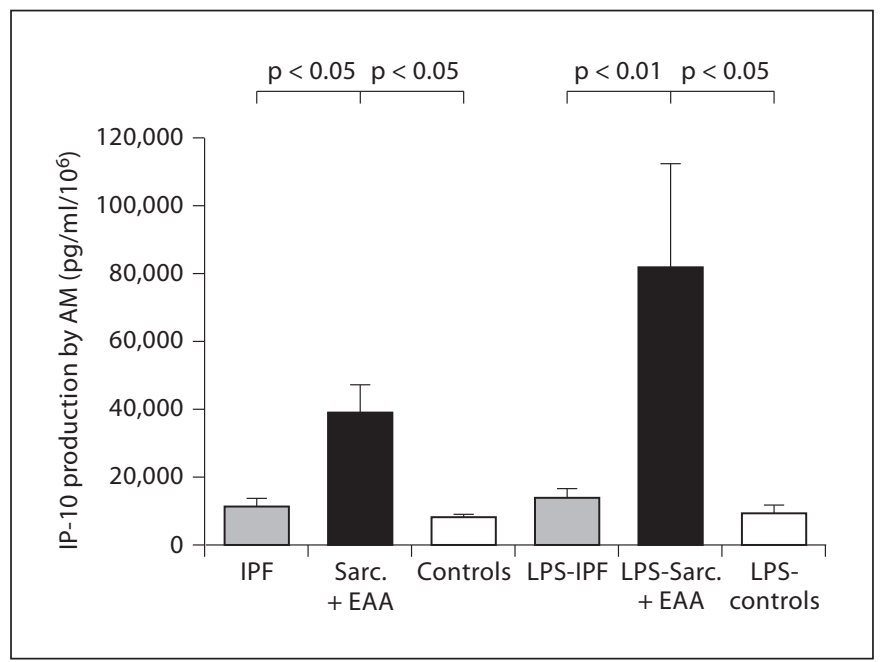

Fig. 3. The release of IP-10 by AM cultured in the absence or presence of LPS stimulation. Data are mean \pm SEM. Sarc. = Sarcoidosis.

there was a significant correlation between IP-10 and the percentage or number of lymphocytes $(\mathrm{r}=0.7266, \mathrm{p}<$ $0.01 ; \mathrm{r}=0.6930, \mathrm{p}<0.01$, respectively; fig. 7 ), and a similar significant correlation between MIG and the percentage or number of lymphocytes $(\mathrm{r}=0.6438, \mathrm{p}<0.05 ; \mathrm{r}=$ $0.6975, \mathrm{p}<0.05$, respectively). There were no other correlations between IP-10 or MIG and BAL cell types.

\section{Discussion}

This study showed that the production of distinct CXC chemokines by AM is increased in patients with IPF and granulomatous lung diseases. In IPF patients, IL-8/ CXCL8, a ELR+ CXC chemokine, was increased and correlated with the percentage and absolute number of neutrophils in BAL fluid, whereas the levels of MIG/CXCL9 and IP-10/CXCL10, two ELR- CXC chemokines, were normal. In sarcoidosis and EAA patients, IL-8, MIG and IP-10 were all increased. The levels of MIG and IP-10 correlated with IL-18, a Th1 cytokine, and the percentage and number of lymphocytes in BAL fluid. Although the baseline release of the chemokines was lower in IPF patients, the relative increase after LPS exposure was similar between IPF and granulomatous lung disease patients, indicating that the capacity of the AM to respond to a nonspecific inflammatory stimulus was similar in both disease groups. 


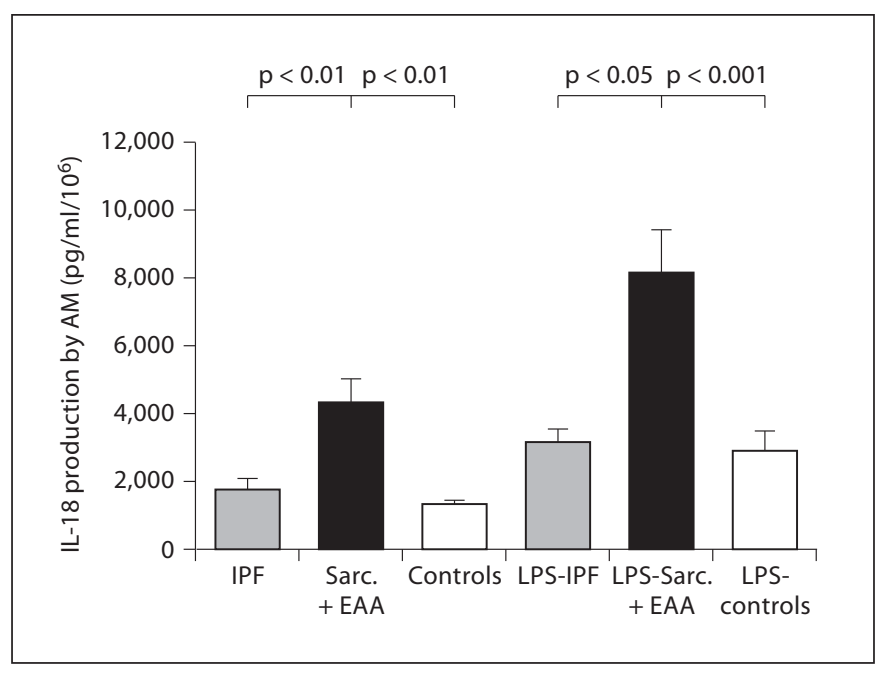

Fig. 4. The release of IL-18 by AM cultured in the absence or presence of LPS stimulation. Data are presented as mean \pm SEM. Sarc. $=$ Sarcoidosis.

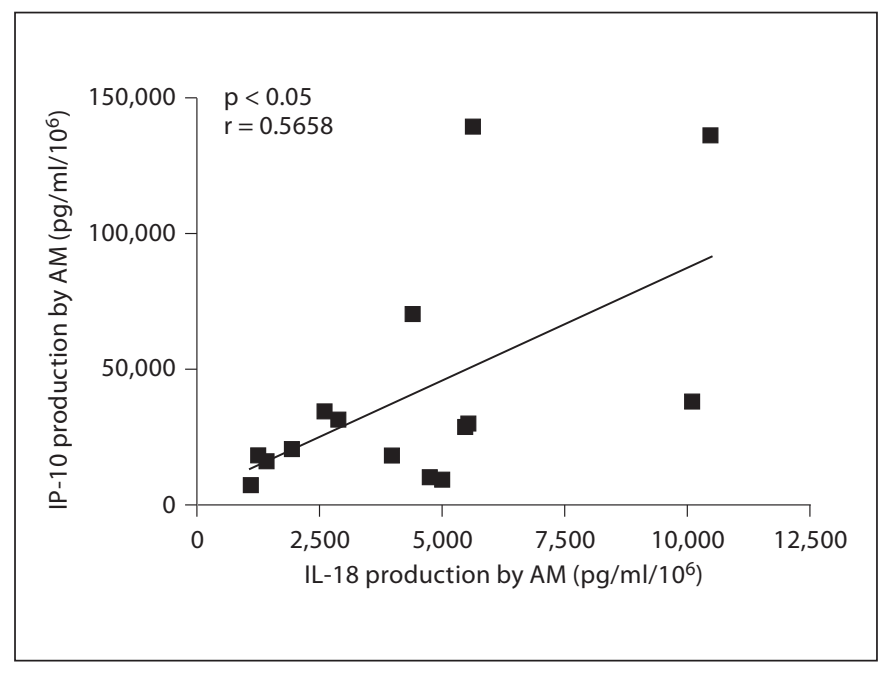

Fig. 5. Correlation between spontaneous IP-10 and IL-18 production by $\mathrm{AM}$ in granulomatous lung diseases.

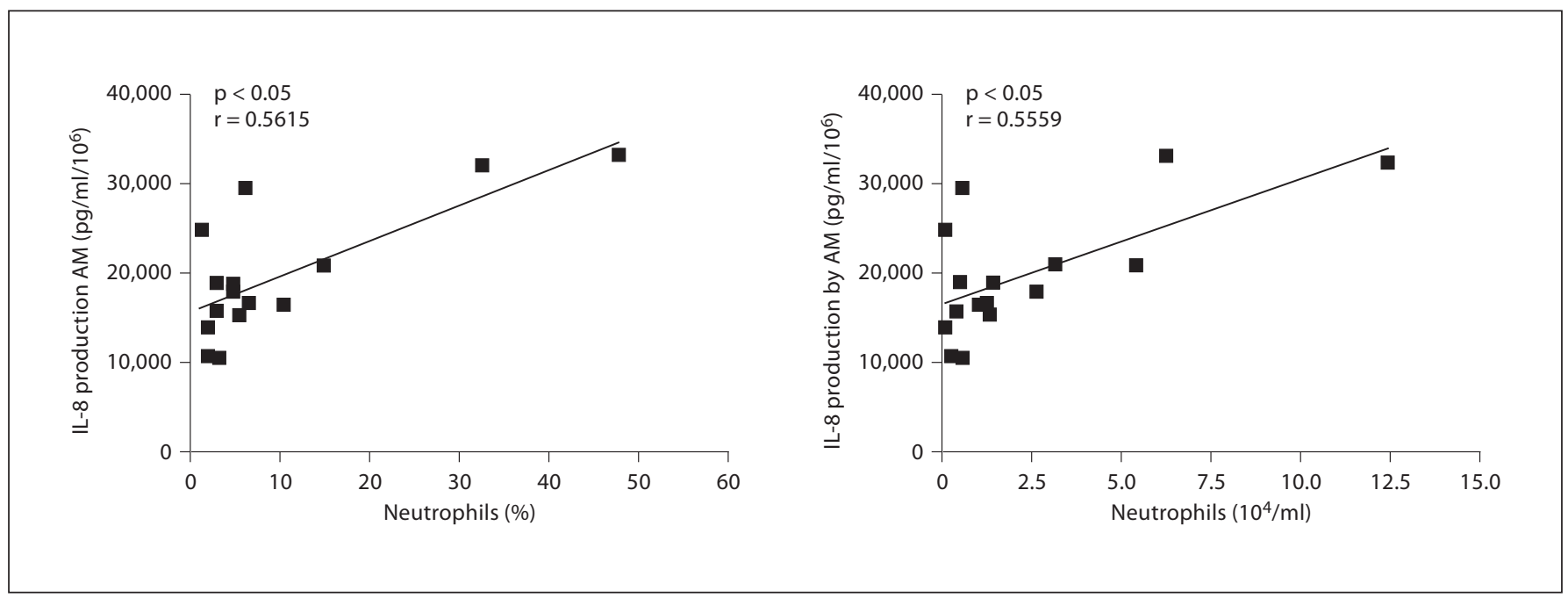

Fig. 6. Correlation between spontaneous IL-8 production by AM and BAL neutrophil percentage and absolute number in IPF patients.

It is known from previous studies that IL-8 levels are increased in serum, BAL fluid, and lung tissue in patients with interstitial lung diseases, including IPF, sarcoidosis and EAA [14-16]. IL-8 is an important chemokine for neutrophils. Neutrophil accumulation in the alveolar space and neutrophil-mediated injury contribute to abnormal lung repair and interstitial fibrosis [17-18]. The process of neutrophil influx into the alveolar spaces appears to be mediated in part by IL- 8 released by macrophages. Alveolar macrophages from patients with IPF spontaneously release and show enhanced expression of IL-8 $[18,19]$. The degree of neutrophilic alveolitis in IPF has been reported to be reflected by increased serum levels of IL-8 [16]. In this study, we found a positive correlation between both percentage and absolute number of neutrophils and the IL- 8 production by AM from IPF patients, which support these results. The increased production of IL- 8 by AM in patients with IPF and granulomatous lung diseases compared to controls may contribute to lung injury and fibrosis formation. 


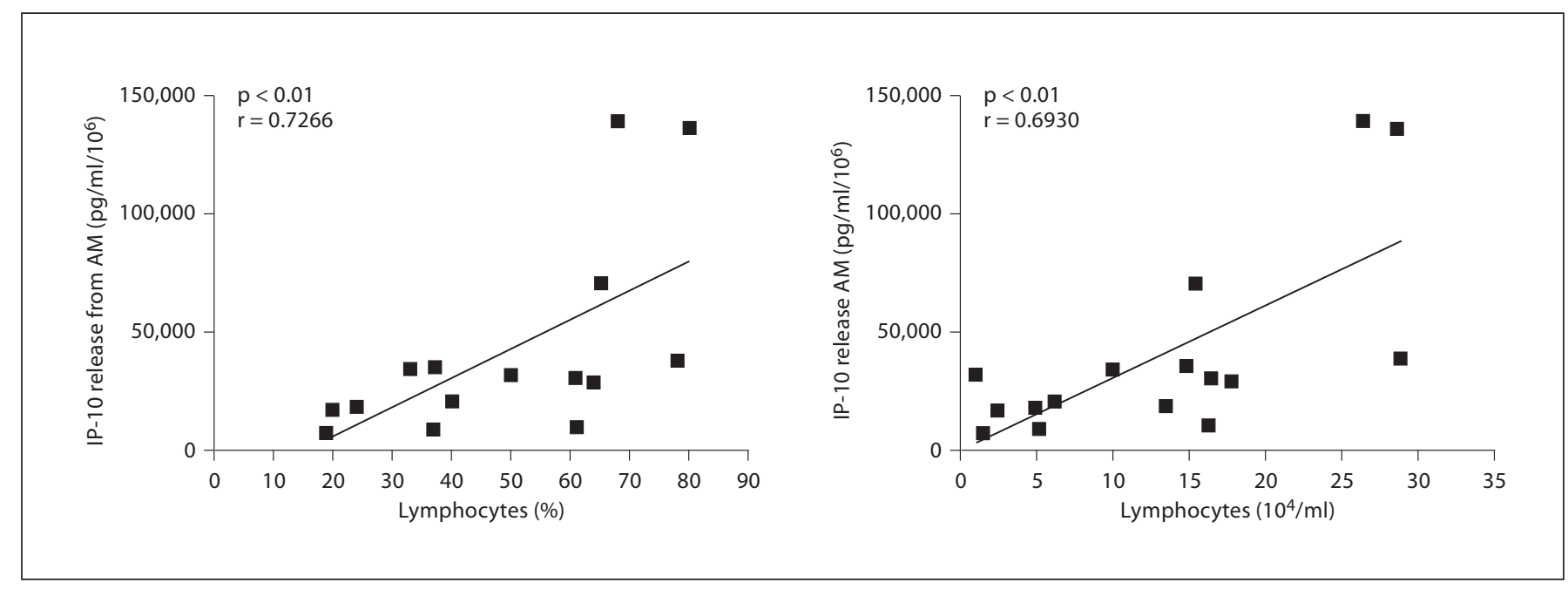

Fig. 7. Correlation between spontaneous IP-10 production by AM and BAL lymphocyte percentage and absolute number in granulomatous patients.

MIG and IP-10 are two ELR- CXC chemokines. Increased serum levels of MIG and IP-10 have been observed in ocular sarcoidosis [20]. Increased production of MIG and IP-10 in the lungs has also been demonstrated during extrinsic allergic alveolitis in mice [21]. MIG and IP-10 BAL levels have been reported to be significantly higher in sarcoidosis than in IPF patients [22]. IP-10 participates in the formation of sarcoid granulomas. Increased IP-10 protein levels and mRNA expression in BAL fluid and biopsy samples have been demonstrated in pulmonary sarcoidosis $[23,24]$. The potential role of IP-10 to regulate the migration and activation of $\mathrm{T}$ cells, but not neutrophils, towards sites of sarcoid activity has been suggested in sarcoidosis [24]. In this study we found an elevated production of MIG and IP-10 by AM in sarcoidosis and EAA, but not in IPF, and a positive correlation between the levels of these 2 chemokines and the percentage/absolute number of lymphocytes in BAL fluid, providing further evidence for the role of these chemokines in recruiting lymphocytes to the lungs in granulomatous lung diseases.

Angiogenesis, the formation of new vessels from the pre-existing vasculature, is an integral part of the physiologic and pathologic tissue repair responses, and can be beneficial or detrimental depending upon the context. Studies have suggested that angiogenesis might be important in the development of pulmonary fibrosis $[5,6]$. Neovascularization in fibroproliferative disorders is regulated by an opposing balance between angiogenic and angiostatic factors. Molecules that originally promote angiogenesis include ELR+ CXC chemokines, such as IL-
8, whereas ELR-CXC chemokines, such as MIG and IP10 , behave as potent inhibitors of angiogenesis and benefit angiostasis [25]. An imbalance between the expression of pro-angiogenic and angiostatic CXC chemokines, such as an increase in IL-8 and a decrease in IP-10, favoring net angiogenic activity, has been demonstrated in lung fibrosis specimens in IPF [26]. When endogenous macrophage inflammatory protein-2, an ERL+ CXC chemokine, was depleted or exogenous IP-10 was administered to animals during exposure to bleomycin, a marked attenuation of pulmonary fibrosis was observed [27, 28].

However, the role of neovascularization in the pathogenesis of IPF is still controversial. Compared with control lungs, capillary density was found to be increased in IPF lungs in areas of minimal fibrosis, but decreased in the most extensively fibrotic lesions [29]. Decreased levels of vascular endothelial growth factor have been found in BAL fluid and increased levels of potent angiostatic factors in tissue biopsy homogenates of IPF, indicating a net angiostatic predominance [30,31]. Our study showed an increased production of IL-8, an angiogenic ELR+ CXC chemokine, but not of two ELR- CXC chemokines, by $\mathrm{AM}$ in IPF. In contrast, in granulomatous lung diseases, the increased production of ELR+ and ELR- CXC chemokines may contribute to keep the balance between angiogenic and angiostatic activity.

IL-18 is a typical Th1 cytokine which is able to induce IFN- $\gamma$ production. IFN- $\gamma$ can induce ELR- CXC chemokines such as IP-10 and MIG, which inhibit angiogenesis. Therefore, IL-18, via the induction of IFN- $\gamma$, will have a 
profound effect on the production of ELR- CXC chemokines [32]. In the current study, we found a positive correlation between IL-18 and MIG/IP-10 production from $\mathrm{AM}$ in vitro, which is in agreement with the hypothesis that Th1 cytokines can up-regulate MIG and IP-10 production.

A limitation of this study is that age and smoking status were different between IPF and granulomatous disease patients. It is unclear whether this has an effect on the measured cytokines, but only one of the IPF patients was a current smoker, the majority were ex-smokers.

In conclusion, our study provides evidence of increased production of IL-8, but not MIG and IP-10, by
$\mathrm{AM}$ in IPF patients. In contrast, the production of all 3 chemokines, IL-8, MIG and IP-10, by AM was increased in sarcoidosis and EAA. This difference in the expression of CXC chemokines and a Th1 cytokine may contribute to the different immunopathogenesis, clinical course and responsiveness to treatment of these diseases.

\section{Acknowledgment}

This work was supported by AFPR (Arbeitsgemeinschaft zur Förderung der Pneumologie an der Ruhrlandklinik).

\section{References}

1 American Thoracic Society/European Respiratory Society: International multidisciplinary consensus classification of idiopathic interstitial pneumonias: general principles and recommendations. Am J Respir Crit Care Med 2002;165:277-304.

2 Newman LS, Wasfi YS: Sarcoidosis. Prog Respir Res 2007;36:128-138.

$\checkmark 3$ Vogelmeier C: Hypersensitivity pneumonitis. Prog Respir Res 2007;36:139-147.

-4 Singer AJ, Clark RA: Cutaneous wound healing. N Engl J Med 1999;341:738-746.

5 Michael PK, et al: ENA-78 is an important angiogenic factor in idiopathic pulmonary fibrosis. Am J Respir Crit Care Med 2001; 164:2239-2242

6 Peao MND, et al: Neoformation of blood vessels in association with rat lung fibrosis induced by bleomycin. Anat Rec 1994;238:5767.

$\checkmark 7$ Zlotnik A, Yoshie O: Chemokines: a new classification system and their role in immunity. Immunity 2000;12:254-257.

$\checkmark 8$ Strieter RM, et al: The functional role of the ELR motif in CXC chemokine-mediated angiogenesis. J Biol Chem 1995;270:2734827357

99 Gerard C, Rollins BJ: Chemokines and disease. Nat Immunol 2001;2:108-115.

10 Gasperini S, et al: Gene expression and pro duction of the monokine induced by IFNgamma (MIG), IFN-inducible T cell alpha chemoattractant (I-TAC), and IFN-gammainducible protein-10 (IP-10) chemokines by human neutrophils. J Immunol 1999;162: 4928-4937.

-11 Coughlin CM, et al: Interleukin-12 and interleukin-18 synergistically induce murine tumor regression which involves inhibition of angiogenesis. J Clin Invest 1998;101:14411452.
12 American Thoracic Society/European Respiratory Society: Idiopathic pulmonary fibrosis: diagnosis and treatment. International consensus statement. American Thoracic Society (ATS) and the European Respiratory Society (ERS). Am J Respir Crit Care Med 2000;161:646-664.

$\checkmark 13$ Tong ZH, et al: Inhibition of cytokine release from alveolar macrophages in pulmonary sarcoidosis by pentoxifylline: comparison with dexamethasone. Chest 2003;124:15261532.

14 Fujimori Y, et al: The role of interleukin-8 in interstitial pneumonia. Respirology 2003;8 33-40.

15 Car BD, et al: Elevated IL- 8 and MCP-1 in the bronchoalveolar lavage fluid of patients with idiopathic pulmonary fibrosis and pulmonary sarcoidosis. Am J Respir Crit Care Med 1994;149:655-659.

16 Ziegenhagen MW, et al: Serum level of interleukin 8 is elevated in idiopathic pulmonary fibrosis and indicates disease activity. Am J Respir Crit Care Med 1998;157:762-768.

17 Gadek JE, et al: Collagenase in the low respiratory tract of patients with idiopathic pulmonary fibrosis. N Engl J Med 1979;301:737742.

18 Hunninghake GW, Gadek JE, Lawley TJ Mechanisms of neutrophil accumulation in the lungs of patients with idiopathic pulmonary fibrosis. J Clin Invest 1981;68:259-269.

19 Lynch JP 3rd, et al: Neutrophilic alveolitis in idiopathic pulmonary fibrosis: the role of interleukin-8. Am Rev Respir Dis 1992;145: 1433-1143.

20 Takeuchi M, et al: Elevated serum levels of CXCL9/monokine induced by interferon- $\gamma$ and CXCL10/interferon- $\gamma$-inducible protein-10 in ocular sarcoidosis. Invest Ophthalmo Vis Sci 2006;47:1063-1068.

-21 Nance S, Cross R, Fitzpatrick E: Chemokine production during hypersensitivity pneumonitis. Eur J Immunol 2004;34:677-685.
-22 Antoniou KM, et al: Different angiogenic activity in pulmonary sarcoidosis and idiopathic pulmonary fibrosis. Chest 2006;130: 982-988

23 Miotto D, et al: Expression of IFN-gammainducible protein, monocyte chemotactic proteins 1, 3, and 4, and eotaxin in TH1- and TH2-mediated lung diseases. J Allergy Clin Immunol 2001;107:664-670.

24 Agostini C, et al: Involvement of the IP-10 chemokine in sarcoid granulomatous reactions. J Immunol 1998;161:6413-6420.

25 Strieter RM, et al: CXC chemokines in angiogenesis. Cytokine Growth Factor Rev 2005; 16:593-609.

26 Keane MP, et al: The CXC chemokines, IL-8 and IP-10, regulate angiogenic activity in idiopathic pulmonary fibrosis. J Immunol 1997;159:1437-1443

$\checkmark 27$ Keane MP: Neutralization of the CXC chemokine, macrophage inflammatory protein2, attenuates bleomycin-induced pulmonary fibrosis. J Immunol 1999; 162:5511-5518.

$>28$ Keane MP: IFN-gamma-inducible protein10 attenuates bleomycin-induced pulmonary fibrosis via inhibition of angiogenesis. J Immunol 1999;163:5686-5692.

$\checkmark 29$ Ebina $M$, et al: Heterogeneous increase in CD34-positive alveolar capillaries in idiopathic pulmonary fibrosis. Am J Respir Crit Care Med 2004;169:1203-1208.

30 Meyer KC, Cardoni A, Xiang ZZ: Vascular endothelial growth factor in brochoalveolar lavage from normal subjects and patients with diffuse parenchymal lung disease. J Lab Clin Med 2000;135:332-338.

31 Cosgrove GP, et al: Aberrant angiogenesis in idiopathic pulmonary fibrosis (abstract). Am J Respir Crit Care Med 2003;167:A300.

32 Robert MS, John AB, Michael PK: CXC chemokines in angiogenesis related to pulmonary fibrosis. Chest 2002;122:298-301. 